\title{
Iperparatiroidismo, Calciomimetici e Trapianto renale
}

\section{S. Bandini}

\author{
Nefrologia dei Trapianti e Dialisi, Azienda Ospedaliera Universitaria Careggi, Firenze
}

\section{Introduzione}

Il trattamento dell'osteopatia del paziente trapiantato renale, rappresenta ancor oggi un problema di difficile soluzione: il trapianto renale, infatti corregge solo parzialmente l'osteodistrofia renale e oltre il 90\% dei pazienti trapiantati renali ha evidenza istologica di osteopatia. Varie sono le "noxae" patogene presenti nell'osso del paziente trapiantato in parte "ereditate" dagli anni trascorsi in dialisi, in parte acquisite col trapianto renale (terapia immunosoppressiva). In estrema sintesi le forme più frequenti di patologia ossea che il nefrologo deve affrontare sono:

1. l'iperparatiroidismo persistente associato o meno a ipercalcemia;

2. la sindrome osteopenica osteoporotica e le fratture secondarie alla patologia osteoporotica, queste ultime, complicanze ossee più strettamente correlate al trapianto.

Mi limiterò in questo articolo ad affrontare il problema del trattamento chirurgico e/o farmacologico dell'iperparatiroidismo (IPTS) residuale ipercalcemico del trapiantato renale, alla luce della disponibilità di una nuova opzione terapeutica: i calciomimetici.

\section{Iperparatiroidismo persistente}

Un trapianto renale ben funzionante, determina un graduale ripristino della sintesi di calcitriolo, una riduzione dei livelli di fosforemia con comparsa di franca ipofosfatemia che perdura nel tempo e spesso, una assai più lenta normalizzazione della funzione paratiroidea. Alcuni autori riferiscono di una normalizzazione dei livelli di PTH in tempi brevi (3 mesi), altri docu- mentano in una larga percentuale di pazienti (30-50\%), livelli di PTH persistentemente elevati per anni (1-3). Lobo (4) in uno studio su 52 pazienti con intatta funzione del rene trapiantato, rileva che, ad un anno dal trapianto, oltre il 50\% dei pazienti presentano livelli di PTH al di fuori del range di normalità. Torres (5), ha evidenziato in uno studio similare, che dopo circa 3 anni dal trapianto, l' $80 \%$ dei pazienti con graft ben funzionante, era iperparatiroideo. Lo stesso autore ha mostrato che esiste un nesso causale tra un non adeguato controllo delliperparatiroidismo secondario pre-trapianto e persistenza dell'osteopatia post-trapianto. Montalban (6) osserva che nel $40 \%$ dei pazienti trapiantati da circa 10 anni, e con un VFG normale, persiste una aumentata secrezione di ormone paratiroideo. Tutto ciò è la conseguenza di un iperparatiroidismo cosiddetto residuale, associato frequentemente a ipercalcemia, che è l'espressione di vari fattori: 1. una lenta involuzione del tessuto paratiroideo iperplastico che può richiedere molti anni;

2. presenza o meno di un quadro di iperplasia nodulare; 3. l'età dialitica pretrapianto;

4. ridotta funzione dell'organo trapiantato: pazienti con graft non ben funzionante e pazienti che a seguito di un rigetto cronico o eventi infettivi sviluppano tardivamente una insufficienza renale. L'ipercalcemia oltreché secondaria al grado di iperplasia paratiroidea, può essere la conseguenza di un aumentato riassorbimento di calcio dai depositi di fosfato di calcio presenti nei tessuti molli o l'espressione di una adeguata sintesi renale di calcitriolo che attiva l'osteolisi osteoclastica da parte dell'ormone paratiroideo o stimola direttamente un aumentato riassorbimento intestinale e tubulare di calcio. 
In alcuni casi, nei primi giorni dal trapianto, si può verificare un grave iperparatiroidismo ipercalcemico (livelli di calcemia $>12-13 \mathrm{mg} / \mathrm{dL}$ ), associato a mancata ripresa funzionale dell'organo trapiantato e a calcificazioni sottocutanee diffuse su tutta la superficie corporea. Tali eventi assai rari, sono espressione di un non adeguato controllo farmacologico di un iperparatiroidismo severo pretrapianto. È opportuno pertanto non inserire o mantenere in lista di trapianto, pazienti che non riescano a mantenere condizioni stabili di euparatiroidismo se non ricorrendo a trattamenti farmacologici aggressivi (dosi elevate di vitamina $\mathrm{D}$ associate a calciomimetici): una paratiroidectomia subtotale, se efficace, mette al sicuro il trapiantando da questa temibile complicanza.

Molto più frequentemente il nefrologo deve trattare un iperparatiroidismo persistente ipercalcemico che se non corretto, può provocare nel tempo, oltreché una accentuata demineralizzazione ossea, un progressivo deterioramento della funzionalità dell'organo trapiantato (oltre il 70\% dei reni trapiantati di soggetti iperparatiroidei presentano calcificazioni interstiziali) e calcificazioni vascolari diffuse con rischio di aumentata incidenza di mortalità cardiovascolare (7). Purtroppo, il trattamento farmacologico tradizionale di questo iperparatiroidismo residuale nel paziente trapiantato, pone al nefrologo problemi a volte di non facile soluzione:

1. Non è proponibile in questi pazienti somministrare vitamina $\mathrm{D}$ e supplementi di calcio per la persistenza degli elevati livelli calcemici.

2. Il trattamento con i difosfonati, utile in teoria, per controllare i livelli calcemici, può, nel tempo determinare un quadro di malattia adinamica dell'osso, soprattutto se si rende indispensabile una paratiroidectomia per trattare l'iperparatiroidismo residuale. Tali farmaci infatti, inibiscono l'osteolisi osteoclastica e tendono ad accumularsi nel tessuto osseo riducendone il turnover.

3. La supplementazione di fosforo per correggere l'ipofosforemia dovuta a una eccessiva escrezione urinaria di fosfati, può esacerbare l'iperparatiroidismo esercitando uno stimolo sulla sintesi di PTH, riducendo i livelli di calcitriolo, e della calcemia $(8,9)$.

4. La possibile dissociazione tra livelli di PTH e istologia ossea può rendere difficile una diagnosi certa di malattia ossea. Uno studio bioptico (10), effettuato su 17 pazienti trapiantati renali con iperparatiroidismo persistente ipercalcemico, ha evidenziato all' istomorfometria ossea che $8 / 17$ pazienti presentavano un reperto istologico patognomonico di una malattia adinamica dell'osso. Pertanto l'autore suggeriva di eseguire una biopsia ossea prima di iniziare una qualunque terapia. Tale suggerimento è ovviamente difficilmente praticabile, ma induce il nefrologo a una attenta valutazione clinico laboratoristica del paziente da trattare e limita ulteriormente l'indicazione a una eventuale paratiroidectomia.

Un iperparatiroidismo persistente associato o meno a ipercalcemia presenta, pertanto, modalità terapeutiche diverse.

\section{Iperparatiroidismo persistente non ipercalcemico}

\section{- Somministrazione di Calcio e Vitamina D}

La vitamina $\mathrm{D}$ e i suoi analoghi sono potenzialmente utili per controllare l'iperparatiroidismo secondario, ma spesso non raggiungono l'effetto perché, anche se inizialmente non presente, favoriscono la comparsa di una ipercalcemia. Comunque, la somministrazione di vitamina $\mathrm{D}$ da sola o con supplementi di calcio può essere utile nel ridurre gli effetti osteopenizzanti dei glucocorticoidi determinando un aumentato assorbimento di calcio (intestinale e renale) e una riduzione della secrezione di PTH. È stato dimostrato, infatti, che nei pazienti trapiantati renali è presente in larga percentuale $(80 \%)$ un deficit di vitamina D (11). E consigliabile, pertanto, la somministrazione di analoghi della vitamina $\mathrm{D}$ : alfacalcidolo, doxercalciferolo (non in commercio in Italia) o calcitriolo (0.25-0.5 $\mu \mathrm{g} /$ die o a gg. alterni). In particolare la somministrazione di alfacalcidolo in pazienti trattati con steroidi ha prevenuto la demineralizzazzione ossea e l'incidenza di fratture (12). In uno studio, in doppio cieco controllato vs placebo, la somministrazione di calcitriolo associato a supplementi di calcio ha ridotto la perdita di massa ossea post trapianto (13). In una review recente, altri autori hanno rilevato che pazienti trapiantati renali trattati con vitamina $\mathrm{D}$ e supplementi di calcio presentavano dopo 6 mesi, un incremento della BMD, mentre, nel gruppo di pazienti di controllo, si osservava un decremento costante nel tempo della BMD (14).

\section{Iperparatiroidismo persistente ipercalcemico}

\section{- Paratiroidectomia \\ - Uso di calciomimetici}

La paratiroidectomia non è esente da problemi e prima di porne l'indicazione, bisogna considerare che sono 
stati segnalati eventi avversi: riduzione della funzione renale, ipocalcemia postoperatoria resistente alla terapia sostitutiva, comparsa di una patologia a basso turnover associata a incrementato rischio di fratture (15). L'ormone paratiroideo, infatti, ha un ruolo centrale nel processo di formazione dell'osso attivando il turnover osseo e frenando parzialmente l'effetto pro-apoptotico degli osteoblasti indotto dagli steroidi. Rojas (16) in un interessante studio, in cui ha effettuato biopsia ossea pre- e post-trapianto, ha rilevato una correlazione positiva tra elevati livelli di PTH pre- e post-trapianto e numero degli osteoblasti: pertanto il PTH potrebbe svolgere un ruolo protettivo sulla sopravvivenza delle cellule stesse.

L'intervento di paratiroidectomia, quindi, non deve provocare un ipoparatiroidismo persistente nel paziente trapiantato renale.

Sono riferiti in letteratura come accennato in precedenza, casi di deterioramento della funzione renale o addirittura di "danno" irreversibile dell'organo trapiantato secondario a paratiroidectomia (17). Rostaing (18) rileva, dopo PTX, un aumento dei livelli di creatinina (oltre il 30\%). Non sono chiarite però le cause della ridotta funzione renale: alcuni studi riferiscono di un incremento di episodi di rigetto renale post PTX, altri autori non confermano questa ipotesi $(8,15)$. Il danno renale sembra correlare con l'entità del decremento dei livelli sierici dell'ormone paratiroideo. Si è ipotizzato un danno funzionale: il PTH ha infatti, una azione favorente la vasodilatazione delle arteriole preglomerulari e una sua brusca riduzione, post PTX potrebbe determinare una insufficienza renale funzionale (19). Questi dati non sono confermati da Jespersen e Malfelder $(20,21)$, che addirittura evidenziano come l'infusione di ormone paratiroideo intrarenale in reni di ratto aumenta il filtrato glomerulare e la diuresi senza modificare la frazione di filtrazione. Oltreché sulla funzione renale a breve termine, non è chiarito, in letteratura, se la paratiroidectomia possa influire anche sulla sopravvivenza a lungo termine dell'organo trapiantato. Quale comportamento seguire? Qualora, in questi pazienti con IPTS persistente ipercalcemico, si verifichi una riduzione del VFG non dipendente da altre cause, associata ad alti livelli di PTH e incremento progressivo dei livelli calcemici $(>12.5 \mathrm{mg} / \mathrm{dL})$, è opportuno porre l'indicazione alla paratiroidectomia. Altrimenti, per le considerazioni in precedenza esposte, conviene ritardare l'intervento tenendo presente che un iperparatiroidismo non severo, per lo stimolo esercitato sul turnover osseo dall'ormone, può giocare un ruolo protettivo sulla patologia ossea propria del trapiantato renale.

\section{- Cinacalcet nel trattamento dell'iperparatiroidi- smo del paziente trapiantato renale}

L'utilizzo del Cinacalcet nei pazienti in dialisi, iperparatiroidei è ampiamente diffuso e molti studi hanno confermato la sua efficacia nel ridurre i livelli di PTH e nel controllare gli altri parametri del metabolismo minerale, anche in casi di iperparatiroidismo severo. Il calciomimetico può rappresentare, altresì, il farmaco ideale (in Italia esiste per ora solo l'indicazione per il trattamento dell'IPTS del paziente uremico e dell'IPTS Primitivo in dialisi) nel trattamento delliperparatiroidismo persistente del paziente trapiantato renale. L'efficacia di tale farmaco pare provata da alcuni studi clinici prospettici e retrospettivi che hanno però il limite di casistiche limitate e di brevi periodi di followup (durata degli studi da 3 a 18 mesi) (22-30). Le caratteristiche dei pazienti scrinati sono riportate nella Tabella I.

Nella Tabella II sono riportati i risultati ottenuti con Cinacalcet nei vari studi.

In tutti gli studi $(23-26,29,30)$ il calciomimetico era somministrato in monoterapia, non associato alla vitamina D eccetto che nel lavoro di Apostolou $(27,28)$, nel quale la vitamina è stata utilizzata, una volta normalizzati i livelli calcemici.

Per lo più i pazienti presentavano una funzione renale stabile, ma con ampie oscillazioni della creatinina clearance $(30-75 \mathrm{~mL} / \mathrm{min})$. L'obiettivo primario della terapia con Cinacalcet era il controllo della ipercalcemia: obiettivo raggiunto dai vari autori con un dosaggio medio del farmaco assai basso $(30-40 \mathrm{mg} /$ die). I livelli calcemici si mantenevano nel range di normalità durante il periodo di osservazione. In alcuni casi la correzione della calcemia era solo parziale (27).

Nei diversi studi, entro 2-4 settimane di trattamento anche i livelli di PTH si riducevano significativamente e tale controllo perdurava nel tempo (10 settimane14 mesi) ( 22, 25-27) (Tab. II). In particolare Leca (25) ha evidenziato una riduzione del PTH del 40\% rispetto al basale durante il $1^{\circ}$ mese di trattamento; tale decremento si è mantenuto nei 6 mesi di follow-up. Similmente Serra (23) ha osservato una riduzione massimale dell'ormone di oltre il 50\% nelle prime 8 settimane di terapia con Cinacalcet e un controllo adeguato del PTH, nei mesi di follow-up. Lo stesso autore (31), in un recente lavoro, ha evidenziato come esista una correlazione altamente significativa tra le concentrazioni plasmatiche di Cinacalcet e i livelli sierici di PTH.

Nello studio di Kruse (24) il decremento dell'IPTSH non risulta essere significativo per l'ampia variabilità 
TABELLA I - CARATTERISTICHE DEI PAZIENTI

\begin{tabular}{|c|c|c|c|c|c|c|c|c|}
\hline & Serra & Kruse & Schwarz & Leca & Srinivas & Apostolou & Apostolou & El-Amm \\
\hline \multicolumn{9}{|c|}{ Età (anni) } \\
\hline Media & NR & NR & 52 & NR & NR & NR & 62 & 45 \\
\hline \multicolumn{9}{|l|}{ Sesso } \\
\hline Uomo & 6 & 7 & 9 & NR & 6 & 4 & 2 & 8 \\
\hline Donna & 6 & 7 & 0 & NR & 5 & 3 & 0 & 10 \\
\hline \multicolumn{9}{|c|}{$\begin{array}{l}\text { Durata dialisi prima } \\
\text { dello studio (mesi) }\end{array}$} \\
\hline Media & 32 & NR & NR & 65 & NR & NR & NR & 84 \\
\hline \multicolumn{9}{|c|}{ Tempo dal trapianto (mesi) } \\
\hline Media & 28 & NR & 59 & NR & NR & NR & NR & NR \\
\hline Range & $6-384$ & $7-168$ & NR & NR & $2-60$ & $4-35$ & $10-16$ & $1-276$ \\
\hline \multicolumn{9}{|c|}{ PTH tempo 0 (pg/mL) } \\
\hline
\end{tabular}

NR : non riportata (Modificata da Choncol M. NDT 2008)

TABELLA II - RISULTATI TERAPIA CON CALCIOMIMETICO

\begin{tabular}{|c|c|c|c|c|c|c|c|c|}
\hline Studio & Durata (mesi) & Pazienti Cinacalcet & PTH & $\mathbf{C a}$ & $\mathbf{P}$ & $\mathrm{Ca} \times \mathbf{P}$ & Immuno-soppressori & $\begin{array}{c}\text { Funzione } \\
\text { Graft }\end{array}$ \\
\hline Serra & 6 & 12 & $\downarrow$ & $\downarrow$ & $\uparrow$ & $\leftrightarrow$ & $\leftrightarrow$ & $\leftrightarrow$ \\
\hline Schwarz & 6 & 9 & $\downarrow$ & $\downarrow$ & $\uparrow \mathrm{ns}$ & $\leftrightarrow$ & $\leftrightarrow$ & $\leftrightarrow$ \\
\hline Leca & 6 & 10 & $\downarrow$ & $\downarrow$ & $\uparrow \mathrm{ns}$ & NR & NR & NR \\
\hline Apostolou & 14 & 2 & $\downarrow \mathrm{ns}$ & $\downarrow \mathrm{ns}$ & $\uparrow \mathrm{ns}$ & NR & $\leftrightarrow$ & $\leftrightarrow$ \\
\hline El-Amm & 6 & 18 & $\downarrow$ & $\downarrow$ & $\uparrow$ & $\leftrightarrow$ & $\leftrightarrow$ & $\downarrow$ \\
\hline Totale & & 83 & & & & & & \\
\hline
\end{tabular}

ns: non significativo (Modificata da Choncol M. NDT 2008)

dei livelli di PTH dei soggetti esaminati (Tab. II).

Boulanger, contraddicendo i risultati positivi degli studi descritti, segnala, in pazienti con IPTS severo posttrapianto renale, la mancata efficacia del calciomimetico nel controllare i livelli di PTH e della calcemia nonostante dosi elevate del farmaco $(180 \mathrm{mg} / \mathrm{die})$ (32).

La fosforemia incrementa o addirittura si normalizza in 6 studi su 8 (Tab. II). Serra (23) rileva un controllo ottimale dei livelli di fosforemia nel $90 \%$ dei pazienti trattati con Cinacalcet per almeno 6 mesi. In uno studio, assai recente, lo stesso autore (33), chiarisce come il calciomimetico raggiunga tale obiettivo. Nelle prime 8 ore dalla somministrazione, il calciomimetico incrementa in modo dose-dipendente i livelli della fo- sforemia, determinando una contemporanea riduzione della escrezione frazionale del fosforo e dei livelli di PTH. La riduzione della fosfaturia si correla significativamente con i bassi livelli di PTH e non con i livelli di FGF23 che, per contro, rimangono elevati. Da ciò deriva che l'azione fosfatemica del Cinacalcet appare essere PTH dipendente (33).

Nei lavori citati, il prodotto $\mathrm{Ca} \times \mathrm{P}$, se valutato, non si modifica significativamente.

Nonostante questi buoni risultati, non è ancora chiarito, se l'efficacia del calciomimetico nel controllo dei vari parametri del metabolismo calcio-fosforo permane dopo la sospensione del trattamento: cioè per quanto tempo deve essere continuata la somministrazione del calciomimetico, e quanto tempo occorre per 
normalizzare i livelli di PTH nei casi più resistenti alla terapia. Alcuni autori hanno osservato che gli effetti del calciomimetico sul controllo della secrezione paratiroidea persistono, discontinuando o sospendendo il farmaco; ma si tratta di un numero di casi limitato e di follow-up brevi $(34,35)$.

In 2 degli 8 studi, come dopo una PTX, si è verificata una riduzione lieve della funzione renale (Tab. II) $(25,30)$. In uno dei due lavori, il follow-up di soli 3 mesi impedisce di fatto conclusioni su un possibile recupero nel tempo della funzione renale. Va tenuto presente comunque che prima di attribuire al solo Cinacalcet l'effetto sul decremento della funzione renale è opportuno escludere altre cause precedenti o concomitanti che possono interferire quali: infezioni, episodi di rigetto, nefrotossicità da farmaci).

Nei lavori valutati non è stata riferita interazione del Cinacalcet con i farmaci immunosoppressori né un incremento degli episodi di rigetto: alcuni autori, recentemente, hanno suggerito maggiori precauzioni, soprattutto nei pazienti trattati con inibitori della calcineurina $(36,37)$. A questo proposito è opportuno citare uno studio recente di Falck (36) che ha valutato, in 14 pazienti trapiantati renali con stabile funzione renale, l'interazione farmacocinetica tra Cinacalcet, gli inibitori della calcineurina e l'acido micofenolico. Il calciomimetico come è ben noto, dopo somministrazione orale si distribuisce in modo omogeneo nella gran parte dei tessuti; oltre il $95 \%$ è presente in circolo legato alle proteine plasmatiche e dopo ossidazione e coniugazione con acido glicuronico, viene escreto sia per via biliare $(20-40 \%)$ che urinaria $(50 \%)$. Cinacalcet viene metabolizzato dagli enzimi microsomiali CYP1A2 e CYP3A presenti a livello epatico. Gli inibitori della calcineurina (ciclosporina e tacrolimus) utilizzano la stessa via metabolica tramite il CYP3A; pertanto non può essere esclusa una interazione farmacologia fra questi farmaci. Falck et al (37) hanno dimostrato che il Cinacalcet ha un moderato effetto, dopo una settimana di trattamento, sulla farmacocinetica del Tacrolimus, inducendo una riduzione dei livelli sierici dell'immunosoppressore. Il calciomimetico, per contro, non avrebbe alcun influenza su ciclosporina e acido micofenolico. Gli autori concludono che non sembrano necessari aggiustamenti della dose di tacrolimus, ma è opportuna precauzione monitorarne attentamente i livelli plasmatici. Altri studi di farmacocinetica hanno evidenziato che bisogna porre attenzione a somministrare alte dosi di Cinacalcet per l'effetto dose dipendente del calciomimetico sulla calciuria che, se elevata, può interferire sulla funzione renale, determinando nefrocalcinosi
(38). La calciuria aumenta nelle prime 8 ore dalla somministrazione di Cinacalcet: la riduzione della posologia del farmaco, tende a normalizzare l'escrezione urinaria del calcio (39). Il calciomimetico potrebbe essere pertanto, responsabile del ridotto assorbimento tubulare di calcio sia direttamente per l'azione sui recettori per il calcio (CaSR) presenti nel tratto spesso ascendente dell'ansa di Henle (la loro stimolazione provoca una aumentata escrezione frazionaria renale di calcio), sia indirettamente inibendo la secrezione di PTH e quindi il suo effetto anticalciurico. La ipercalciuria anche se transitoria potrebbe favorire una riduzione della densità minerale ossea. A questo proposito vi sono recenti studi che descrivono l'effetto di Cinacalcet anche sulla BMD $(40,41)$. In particolare Campistol (41) ha rilevato in 9 pazienti trapiantati renali un incremento della BMD dopo 12 mesi di trattamento con calciomimetico a dosi variabili. Un miglior controllo dei livelli sierici di IPTSH o una diretta azione del calciomimetico sull'osso potrebbero spiegare l'effetto di Cinacalcet sulla mineralizzazione ossea (41).

Kuzendorf (15) in un suo interessante editoriale affronta il problema delle conseguenze di una discontinuazione di Cinacalcet, nel paziente dializzato, subito prima di un trapianto renale. La farmacocinetica del calciomimetico, che determina una inibizione della secrezione di PTH variabile nelle 24 ore (massimale nelle prime 2-6 ore e progressivamente ridotta nelle 24 ore successive) induce a somministrare il farmaco senza interruzioni, prima e subito dopo il trapianto. L'alternativa, è una recidiva dell'iperparatiroidismo: alla sospensione del Cinacalcet fa seguito infatti, un rapido incremento dei livelli di PTH.

Kamar (42) infine ha dimostrato, in 11 pazienti trapiantati renali, che Cinacalcet può esere somministrato anche due volte al giorno per un lungo periodo di tempo (un anno) senza perdita di efficacia.

\section{Conclusioni}

I risultati di questi studi mostrano che il trattamento con Cinacalcet nei soggetti trapiantati renali è efficace nel normalizzare l'ipercalcemia, nel ridurre i livelli sierici dell'IPTSH e nell'incrementare i livelli sierici del fosforo senza interferire sulla funzionalità del graft. Per confermare questi dati, sono ovviamente indispensabili ulteriori indagini con casistiche più numerose $\mathrm{e}$ follow-up a lungo termine che confermino l'efficacia e la sicurezza del calciomimetico. Uno studio recentis- 
simo di Meola (43) sul controllo, mediante Cinacalcet, della iperplasia paratiroidea di pazienti uremici con severo iperparatiroidismo, può rappresentare uno stimolo ulteriore alla somministrazione prolungata nel tempo del farmaco.
Indirizzo degli Autori:

Sandro Bandini, MD

SOD Nefrologia dei Trapianti e Dialisi

Azienda Ospedaliera Universitaria Careggi

Viale Pieraccini 18

50100 Firenze

sandro.bandini1@ virgilio.it

\section{Bibliografia}

1. Lewin E. Involution of the parathyroid glands after renal transplantation. Curr Opin Nephrol Hypertens 2003; 12: 363-71.

2. Traindl O, Langle F, Reading S, et al. Secondary hyperparathyroidism and acute tubular necrosis following renal transplantation. Nephrol Dial Transplant 1993; 8: $73-6$.

3. Messa PG, Sindici C, Cannella G. Persistent hyperparathyroidism after renal transplantation. Kidney Int 1998; 54:1704-13.

4. Lobo PI, Cortez MS, Stevenson W et al. Normocalcemic hyperparathyroidism associated with relatively low 1,25 vitamin D levels post renal transplant can be successfully treated with oral calcitriol. Clin Transplant 1995; 9: 277-81.

5. Torres A, Rodriguez AP, Concepcion MT, et al. Parathyroid function in long term transplant patients : importance of pretransplant PTH concentrations. Nephrol Dial Transplant 1998; 13 (suppl 3) : 94-97.

6. Montalban M, De Francisco AL, Marinoso ML, Zubimendi JA, Arias M. Bone disease in long term adult kidney transplant patients with normal renal function. Kidney Int 2003; 8(Suppl): S1129 -32.

7. Parfitt AM. Hypercalcemic hyperparathyroidism following renal transplantation: differential diagnosis, management and implications for cell population control in the parathyroid gland. Miner Electrolyte Metab 1999; 8: 92112.

8. Chonchol M, Wüthrich RP. Potential future uses of calciomimetics with chronic kidney disease. Nephrol Dial Transplant 2008; 1(Suppl 1): 36-40.

9. Caravaca F, Fernandez MA, Ruiz-Calero, et al. Effects of oral phosphorus supplementation on mineral metabolism of renal transplant recipients. Nephrol Dial Transplant 1998; 13: 2605-11.

10. Borchhardt K, Sulzbacher I, Fodinger M, et al. Lowturnover bone disease in hypercalcemic hyperparathyroidism after kidney transplantation. Am J Transplant 2007; 7: 2515-21.
11. Ewers B, Gasbjerg A, Moelgaard C, et al. Vitamin D status in kidney transplant patients: need for intensified routine supplementation. AM J Clin Nutr 2008; 87: 431-7.

12. Josephon MA, Schumm LP, ChiuMY, et al. Calcium and calcitriol prophylaxis attenuates postransplant bone loss. Transplantation 2004; 78: 1233-6.

13. Reginster JY, Lecart MP, Sarlet N, Defraigne JO. Alphacalcidol in prevention of glucocorticoid-induced osteoporosis. Calcif Tissue Int 1999; 65: 328-31.

14. De Sevaux RG, Hoitsma AJ, Cortstens FH, Wetzels JF. Treatment of vitamina $\mathrm{D}$ and calcium reduces bone loss after renal transplantation: a randomized study. J Am Soc Nephrol 2002; 13: 1608-12.

15. Kunzendorf U, Kramer KB, Arns W, et al. Bone disease after renal transplantation. Nephrol Dial Transplant 2008; 23: 450-58.

16. Rojas E, Carlini Rg, Clesca P, et al. The pathogenesis of osteodystrophy after renal transplantation as detected by early alterations in bone remodeling. Kidney Int 2003; 63: 1915-23.

17. Schwarz A, Rustien G, Merkel S, et al. Decreased renal transplant function after parathyroidectomy. Nephrol Dial Transplant 2007; 22: 584-91.

18. Rostaing L, Moreau-Gaudry X, Baron E, et al. Changes in blood pressure and renal function following subtotal parathyroidectomy in renal transplant patients presenting with persistent hypercalcemic hyperparathyroidism. Clin Nephrol 1997; 47: 248-55.

19. Endlich K, Massfelder T, Helwig J. Vascular effects of parathyroid hormone and parathyroid hormone related protein in the split hydronephrotic rat kidney. J Physiol 1995; 483: 481-90.

20. Massfelder T, Parekh N, Endlich K, et al. Effect of intrarenally infused parathyroid hormone-related protein on renal blood flow and glomerular filtration rate in the anaesthetized rat. Br J Pharmacol 1996; 118: 1995-2000.

21. Jespersen B, Randlov A, Abrahamsen J, et al. Effects of PTH on blood pressure, renal function, hormones, in essential hypertension: the altered pattern of reactivity may counteract raised blood pressure. Am J Hypertens 1997; 10: 1356-67. 
22. Serra AL, Schwarz AA, Wick FH, et al. Successful treatment of hypercalcemia with cinacalcet in renal transplant recipients with persistent hyperparathyroidism. Nephrol Dial Transplant 2005; 20: 1315-19.

23. Serra AL, Savoca R, Huber AR, et al. Effective control of persistent hyperparathyroidism with cinacalcet in renal allografts recipients. Nephrol Dial Transplant 2006; 22: 577-83.

24. Kruse AE, Eisenberge U, Frey FJ, et al. The calcimimetic cinacalcet normalizes serum calcium in renal transplant patients with persistent hyperparathyroidism. Nephrol Dial Transplant 2005; 20: 1311-14.

25. Leca N, Lafttavi M, Gundroo A, et al. Early and severe hyperparathyroidism associated with hypercalcemia after renal transplant treated with cinacalcet. Am J Transplant 2006; 6: 2391-95.

26. Swarc I, Argiles A, Garrigue V, et al. Cinacalcet chloride is efficient and safe in renal transplant recipients with post transplant hyperparathyroidism.Transplantation 2006; 82: 675-80.

27. Apostolou T, Damianou L, Kotsiev V, et al. Treatment of severe hypercalcemia due to refractory hyperparathyroidism in renal transplant patients with the calcimimetic agent cinacalcet. Clin Nephrol 2006; 65: 374-77.

28. Apostolou T, Kollia K, Damianou L, et al. Hypercalcemia due to resistant hyperparathyroidism in renal transplant patients treated with the calcimimetic agent cinacalcet. Transplant Proc 2006; 38: 3514-16.

29. Srinivas TR, Schold JD, Womer KL, et al. Improvement in hypercalcemia with cinacalcet after kidney transplantation. Clin J Am Soc Nephrol 2006; 1: 323-26.

30. El-Amm JM, Doshi MD, Singh A, et al. Preliminary experience with cinacalcet use in persistent secondary hyperparathyroidism after kidney transplantation. Transplan-tation 2007; 83: 546-49.

31. Serra AL, Braun SC, Starke A, et al. Pharmacokinetics and pharmacodynamics of Cinacalcet in patients with hyperparathyroidism after renal transplantation. Am J Transplant 2008; 8: 803-10.

32. Boulanger H, Haymann J P, Fouqueray B, et al. Therapeutic failure of cinacalcet in a renal transplant patient presenting hyperparathyroidism with severe hypercalcemia. Nephrol Dial Transplant 2005; 20: 2865.

33. Serra AL, Wuhrmann C, Wuthricht R. Phosphatemic effect of Cinacalcet in Kidney Transplant Recipients with persistent Hyperparathyroidism. Am J Kidney Dis 2008; 6: 1151-57.

34. Kruse AE, Eisenberger U, Felix JF, Moaupt MG. Effect of cinacalcet cessation in renal- transplant recipients with persistent hyperparathyroidism. Nephrol Dial Transplant 2007; 22: 2362-65.

35. Jean G, Chazot C, Charra B. Six cases of successful cinacalcet cessation in hemodialysis patients treated for secondary hyperparathyroidism. Nephrol Dial Transplant 2007; 22: 577-583.

36. Falck Pal, Vethe NT, Asberg A, et al. Cinacalcet's effect on pharmacokinetics of tacrolimus, cyclosporine and mycophenolate in renal transplant recipients. Nephrol Dial Transplant 2008; 23: 1048-53.

37. Maass E, Mueller GA, Heller T, Koziolek MJ. Decrease in serum Tacrolimus level and rise in S creatinine under late addition of Cinacalcet in a Renal Transplant Recipient with Hyperparathyroidism: A case report. Transplantation Proceedings 2007; 39: 3468-70.

38. Esposito L, Rostaing L, Gennero I, Mehrenberger M, Durand D, Kamar N. Hypercalciuria induced by high dose of cinacalcet in a renal transplant recipient. Clin Nephrol 2007; 4: 245-48.

39. Borchhardt KA, Heinzl H, Mayerwöger E, Hörl WH, Haas M, Sunder-Plassmann G. Cinacalcet Increase Calcium Excretion in Hypercalcemic Hyperparathyroidism After Kidney Transplantation. Transplantation 2008; 7: 919-24.

40. Lien YH, Silva AL, Whittmann D. Effects of cinacalcet on bone mineral density in patients with secondary hyperparathyroidism. Nephrol Dial Transplant 2005; 20: 123237.

41. Bergua C, Torregrosa JV, Fuster D, Dalmau AG, Oppheneimer F, Campistol JM. Effect of Cinacalcet on Hypercalcemia and Bone Mineral Density in Renal Transplanted Patients with Secondary Hyperparathyroidism. Transplantation 2008; 3: 413-17.

42. Kamar N, Gennero I,Spataru L et al. Pharmacodynamic effects of cinacalcet after kidney transplantation: once versus twice daily dose. Nephrol Dial Transplant 2008; 23: 3720-26.

43. Meola M, Petrucci I, Barsotti G. Long term treatment with cinacalcet and conventional therapy reduces parathyroid hyperplasia in severe secondary hyperparathyroidism. Nephrol Dial Transplant 2009; 3: 982-89. 